\title{
At a glance
}

Retinal thickness measurements and segmentation performance of four different spectral and time domain OCT devices

Mylonas et al evaluate the reliability of different OCT devices and scanning patterns in the assessment of retinal thickness and segmentation performance in 28 eyes with $\mathrm{nAMD}$ and 10 healthy eyes. Radial scans $(6 \mathrm{~mm})$ were compared between Stratus and Topcon OCT, in addition to raster scans of three SDOCT devices (Topcon, Spectralis, Cirrus). Retinal thickness values were generally higher in SD-OCT analysis. Spectralis SDOCT demonstrated the highest values of central retinal thickness and Topcon OCT raster scans showed the lowest values. The authors conclude that further development of current algorithms is necessary to improve quantification of retinal thickness. See page 1453

\section{Intravitreal pegaptanib for}

proliferative diabetic retinopathy

González et al compared the efficacy of intravitreal pegaptanib (IVP) with panretinal laser photocoagulation (PRP) in the treatment of active proliferative diabetic retinopathy (PDR) in a prospective, randomised, controlled, open label study. Twenty subjects with active PDR were randomly assigned at a 1:1 ratio to receive treatment in one eye either with IVP $(0.3 \mathrm{mg})$ every 6 weeks for 30 weeks or with PRP. All IVP eyes were completely regressed by week 12 , and maintained through week 36 . In the PRP-treated group, at week 36, two eyes demonstrated complete regression, two showed partial regression, and four showed persistent active PDR. Although, IVP produced rapid regression of PDR, which was maintained throughout the study period, on subsequent follow up, most of the IVPtreated eyes required PRP for recurrent PDR. See page 1474

\section{Predicting visual success in macular hole surgery}

Gupta et al identified factors that predict visual success (better than 6/12) following macular hole surgery by retrospectively analysing the data of 133 patients who underwent standard macular hole surgery. Multivariable regression analysis identified that age, preoperative visual acuity and the preoperative maximum macular hole diameter were significant predictors of visual success. The resulting model correctly classified the visual outcome of $80 \%$ of cases. See page $\mathbf{1 4 8 8}$

\section{Cataract surgery and primary IOL} implantation in children (UK and Ireland)

Solebo et al (British Isles Congenital Cataract Interest Group) report on current patterns of practice relating to primary intraocular lens (IOL) implantation in children ( $\leqslant 2$ years old in the UK and Ireland). $76 \%$ of 928 surveyed ophthalmologists replied. $88 \%$ of surgeons used limbal wounds, $80 \%$ manual capsulotomies, $98 \%$ posterior capsulotomies and $100 \%$ hydrophobic acrylic lenses. However, exclusion criteria for primary IOL implantation varied considerably. The authors conclude that there is a need for systematic studies of the outcomes of primary IOL implantation in younger children. See page 1495

\section{Long-term surgical success of Ahmed Valve implant versus trabeculectomy in POAG}

Tran et al compared the long-term intraocular pressure (IOP) outcomes of Ahmed Glaucoma Valve (AGV) implantation to trabeculectomy with mitomycin C (MMC) in 78 POAG patients who underwent AGV implantation with matched 88 POAG patients who underwent trabeculectomy with MMC with a minimum follow-up of 3 years. The 5 -year cumulative probability of success (IOP $<21 \mathrm{~mm} \mathrm{Hg}$ and a reduction of IOP $>15 \%$ from baseline) was not statistically significant between the 2 groups. However, when a greater IOP reduction was desired (IOP $\leqslant 18 \mathrm{~mm} \mathrm{Hg}$ and a reduction of IOP $>20 \%$ from baseline), trabeculectomy with MMC had a higher rate of success. See page 1504

HRT-3 Moorfields reference plane Asaoka et al assessed the effect of the Moorfields Reference Plane on Heidelberg
Retina Tomograph (HRT) rim area repeatability and its effect on progression rates using event analysis. The Moorfields RP was applied to HRT images from 198 ocular hypertensives, acquired over 6 years. The frequency of identified progression using Moorfields RP was 40\% compared with $28 \%$ for the $320 \mathrm{RP}$. The authors conclude that using Moorfields $\mathrm{RP}$ results in greater rates of detection of change. See page 1510

\section{Orbital decompression for Graves' orbitopathy}

Mourits et al analysed data from euthyroid patients with inactive GO who had undergone orbital decompression for disfiguring proptosis from 11 European centres (European Group on Graves' Orbitopathy (EUGOGO)). The swinging eyelid approach was the most popular followed by the coronal and transconjunctival approaches, achieving average proptosis reduction of $5.0 \mathrm{~mm}$. The proptosis reduction was significantly greater than after three-wall than two-wall decompression. Additional fat removal augmented the proptosis reduction. Complications were rare, the most frequent being worsening of motility, occurring more frequently after coronal decompression. See page 1518

\section{Trends in the incidence of conjunctival melanoma in Sweden}

Triay et al studied the time trends (1960 to 2005) in the incidence of conjunctival melanoma in Sweden $(n=170)$. The agestandardised incidence of conjunctival melanoma increased significantly in men from 0.10 cases/million to 0.74 cases/ million and in women from 0.06 cases/ million to 0.45 cases/million. The annual relative change in age-standardised incidence was $16.9 \%$ in men and $19.5 \%$ in women. During the period of study, tumours became smaller and thinner at the time of diagnosis and increasingly arose from parts of the conjunctiva exposed to ultraviolet radiation. See page 1524 